\title{
Beneficios de las intervenciones basadas en la atención plena para el tratamiento de síntomas ansiosos en niños y adolescentes: Metaanálisis
}

\author{
José Gabriel Soriano', María del Carmen Pérez-Fuentes ${ }^{2 *}$ María del Mar Molero- \\ Jurado', José Jesús Gázquez³ , Begoña María Tortosa' y Alba González \\ 'Universidad de Almería \\ ¿Universidad de Almería / Universidad Politécnica y Artística del Paraguay \\ 3Universidad de Almería / Universidad Autónoma de Chile
}

- Recibido: 07 - 10 - 2019 . Aceptado: 28 - 10 - 2019 . Avance Online: 25 - 11 - 2019

RESUMEN: La ansiedad hace referencia al estado de agitación ante la anticipación de una amenaza, es decir, de una situación peligrosa, lo que conlleva a una respuesta emocional que engloba, tanto el nivel cognitivo, como el emocional, el fisiológico, y/o el motor. El objetivo de este trabajo consiste en identificar la relación existente entre los beneficios de la atención plena (mindfulness) y su influencia en los niveles de ansiedad en niños y adolescentes. Tras realizar una búsqueda exhaustiva de artículos científicos publicados desde la creación de las distintas bases de datos, hasta el mes de agosto de 2019, siendo las bases de datos elegidas Web of Science, PsycINFO, PsycArticles, Scopus, CSIC, Psicodoc, y PubMed, se obtuvieron un total de 2025 artículos de carácter científico que, tras aplicar los criterios de inclusión establecidos, quedaron siete artículos para revisión. Los resultados muestran los beneficios de la intervención del mindfulness (MBI) sobre el sujeto. En este sentido, los resultados sugieren que cuando se llevan a cabo intervenciones basadas en conciencia plena se ve relacionado con una reducción de los niveles de ansiedad, y además, un mayor bienestar personal.

PALABRAS CAVE: Mindfulness, Ansiedad, Niños, Adolescentes, Metaanálisis.

Benefits of mindfulness-based symptoms for the treatment of anxious symptoms in children and adolescents: Meta-analysis

ABSTRACT: Anxiety refers to the state of agitation in the face of the anticipation of a threat, that is, of a dangerous situation, which gives rise to an emotional response, which encompasses both the cognitive level, as well as the emotional, physiological and/or motor level. The aim of this work is to identify the relationship between the benefits of mindfulness and its influence on anxiety scores in children and adolescents. After conducting a search of scientific articles published since the creation of the different databases, until august 2019, being the databases used Web of Science, PsycINFO, PsycArticles, Scopus, CSIC, Psicodoc, and PubMed, were obtained a total of 2025 articles of a scientific nature that, after applying the established inclusion criteria, left seven articles for review. Their results show the influence exerted by intervention programs, in this case, mindfulness (MBI). In this sense, the results suggest that when they carry out full-awareness intervention techniques, it is related to a reduction in anxiety scores and, consequently, to the presence of greater personal well-being.

KEY WORDS: Mindfulness, Anxiety, Children, Adolescents, Meta-analysis.

La intervención basada en el mindfulness (MBI) se ha convertido en los últimos años en

\footnotetext{
*Correspondence: María del Carmen Pérez-Fuentes.

Universidad de Almería / Universidad Politécnica y Artística del

Paraguay

CP: 04120 , Almería, España

E-mail:mpf421@ual.es
}

(C) 2020 Sociedad Universitaria de Investigación en Psicología y Salud. Publicado por Consejo General de Colegios Oficiales de Psicólogos, España. Este es un artículo Open Access

Citar como/Cile as: Soriano, J. G. Pérez-Fuentes, M. C., Moro-Jurado, M. M. Citar como/Cite as: Soriano, J. G., Perez-Fuentes, M. C., Molero-Jurado, M. M.,
Gázquez, J. J., Tortosa, B. M. y González, A. (2020). Beneficios de las intervenciones basadas en la atención plena para el tratamiento de síntomas ansiosos en basadas en la atención plena para el tratamiento de síntomas ansiosos en niños y adolescentes: Metaanálisis Revista lberoame 17 (1), 42-53. hittps://doi.org/10.23923/i.rips.2020.01.034 objeto de investigación por diversos autores, como por ejemplo, los trabajos realizados por López-González, Amutio, Oriol, Gázquez, y Pérez-Fuentes (2018) o por Rawlett, Friedmann, y Thomas (2019), llevándose a cabo dichas investigaciones en diversos contextos (Macías, Valero-Aguayo, Bond, y Blanca, 2019; Roldán, Salazar, Garrido, y Cuevas-Toro, 2015), como 
por ejemplo el educativo (Sanger, Thierry, y Dorjee, 2018), el de la salud (Kemper, Mo, y Khayat, 2015), entre otros (Lutz, Brühl, Scheerer, Jäncke, y Herwig, 2016), debido a los beneficios de dicha intervención en la calidad de vida del sujeto (Zeidan et al., 2016). Además, diversos estudios indican que a través de la intervención del mindfulness se reducen los niveles de ansiedad (Foster, Lomas, Downey, y Stougch, 2018) y de estrés (Barrera-Herrera, Neira-Cofré, Raipán-Gómez, Riquelme-Lobos, y Escobar, 2019; Roselló, Zayas, y Lora, 2016), de manera que el sujeto tras dicha intervención presenta un mayor bienestar personal (Giménez y Tortosa, 2018; Virgili, 2015; Gallego, AguilarParra, Cangas, Rosado, y Langer, 2016).

\section{- MINDFULNESS E INFLUENCIA EN EL SUJETO}

El mindfulness se constituye como una estrategia que potencia el aprendizaje hacia la disminución de la evitación, el apego a los pensamientos, así como, el incremento de la propia capacidad para centrarse en el momento presente, tal y como indican Amutio et al. (2018). Es decir, consiste en la práctica de cultivar la mente (Duarte y Pinto-Gouveia, 2016). En la literatura actual, existen diversas aceptaciones en castellano para referirse al término mindfulness, entre las que se encuentran "conciencia plena", "presencia plena" y "atención plena" (Vallejo, 2006), haciendo todas ellas referencia al estado que implica la atención del sujeto en relación a la fenomenología de las vivencias, englobando, tanto aquellos eventos internos, como externos, disminuyendo consigo la tendencia hacia el juicio continuo (Larrubia, 2019). No obstante, existen diversas situaciones que sí justifican por parte del sujeto una conducta ansiosa, como sería por ejemplo el caso de violencia de género (Guerra y Barrera, 2017) o cualquier otro tipo de violencia, puesto que la violencia lleva aparejada respuestas de conductas agresivas ante diversos escenarios (Pérez-Fuentes et al., 2015) y por diversas causas (Gázquez et al. 2016; Gázquez, PérezFuentes, Carrión, Luque, y Molero, 2015; Pérez-Fuentes, Molero, Carrión, Mercader, y Gázquez, 2016), generándose dicha conducta con más frecuencia en las etapas de la adolescencia (Alarcón, Pérez-Luco, Wenger, Salvo, y Chesta, 2018; Álvarez-García, Núñez, García, y Barreiro-Collazo, 2018). De modo que, dichos escenarios llegan a causar un incremento en el nivel de ansiedad del sujeto (Zuñeda, Yamazares, Marañon, y Vázquez, 2016). En este sentido, la práctica de MBI reduce los niveles de ansiedad (Lothes, Hakan, y Kassab, 2013; Strawn et al., 2014), puesto que, los programas de atención contribuyen a reducir dichos niveles (Ho, Tong, Mak, y Kei, 2018).

En algunos niños las actividades escolares diarias presentan una gran cantidad de estresores potenciales. No obstante, ciertos estresores son ciertamente útiles, ya que se constituyen como una respuesta esperada al estrés, pero es cierto que demasiada ansiedad, interfiere con la capacidad del niño para estudiar y hacer lo apropiado para el progreso académico, por ejemplo. En este sentido, la ansiedad se asocia comúnmente con sesgos intencionales (Ehrenreich y Gross, 2002), cognitivos (Semple, Lee, Rosa, y Miller, 2010), y de hiperactivación fisiológica (Joiner et al., 1999). Parece probable que las preocupaciones asociadas con la ansiedad interfieren en lo académico (Duncan et al. 2007). En consecuencia, el mindfulness se ha visto relacionado con otros factores que hacen que mejore la calidad de vida de la persona, como, por ejemplo, en el correcto desarrollo de una aceptación sin prejuicios de la propia percepción de la realidad, incluidas las emociones negativas y el estrés (Bishop et al., 2004). Igualmente, la terapia de presencia plena fomenta también un correcto estilo de alimentación. Es decir, comer en respuesta al hambre fisiológico y no en lugar de señales situacionales y emocionales (Tylka, 2006). Pues, a través del mindfulness, los sujetos no solo vuelven a ser menos receptivos a señales externas de alimentos, de estrés, de emociones negativas o de antojos como desencadenantes de ingesta de alimentos (Tapper, 2017), sino que, aprenden a prestar toda la atención al proceso que conlleva el comer (Warren, Smith, y Ashwell, 2017). 


\section{- MINDFULNESS Y ANSIEDAD}

La ansiedad hace referencia al estado de agitación ante la anticipación de una amenaza, es decir, una situación peligrosa, originando dicho estado, una respuesta emocional, que engloba, tanto el nivel cognitivo, como el emocional, el fisiológico y/o el motor. Pues, la ansiedad constituye una experiencia universal presente en la vida del sujeto, pero cuando esta excede los límites de normalidad puede llegar a ocasionar trastornos (Soto-Vázquez y AlvaradoGarcía, 2018). Así pues, hay que tener en cuenta aquellos factores protectores que permiten minimizar los distintos componentes ambientales que causan situaciones estresantes, como sería el desarrollo de habilidades de afrontamiento, de comunicación, así como de resolución eficaz de problemas (Peña, Bernal, Pérez, Reyna, y García, 2017), entre otros (Gázquez et al., 2019).

Tras analizar la literatura previa, se ha podido comprobar que se han realizado muy pocas intervenciones destinadas a niños y adolescentes, y que tengan como objetivo analizar los niveles de ansiedad de los sujetos tras dichas intervenciones. En este sentido, Biegel, Shapiro, Brown, y Schubert (2009) llevaron a cabo un estudio para analizar diversas variables psicológicas, entre las que se encontraba la ansiedad, intentando estudiar la eficacia de la intervención de MBI sobre las puntuaciones de ansiedad en adolescentes, ya que la práctica mente-cuerpo se ve favorecida por los estados de conciencia (Cuéllar, Bazán, y Araya, 2019). De este modo, la práctica de presencia plena mejora el bienestar de la persona, reduciendo los síntomas de estrés percibido, de ideas negativas (Díaz-González, Pérez, Sánchez-Raya, Moriana, y Sánchez, 2018), así como, de ansiedad y depresión (Liehr y Diaz, 2010), viéndose relacionada con un incremento de la regulación emocional y de la atención plena (Cotton et al., 2016), además de favorecer otros factores (Redstone, 2015; Strawn et al., 2016).

Así, el objetivo de este trabajo consiste en identificar la relación existente entre la intervención en atención plena (mindfulness) y los niveles de ansiedad en niños y adolescentes.

\section{METODOLOGÍA}

\section{-PROCEDIMIENTO}

Se llevó a cabo una búsqueda exhaustiva en distintas bases de datos, siguiendo las recomendaciones PRISMA propuestas por Moher et al. (2009), para así poder establecer conclusiones (Antman, Lau, Kupelnick, Mosteller, y Chalmers, 1992). Las bases de datos consultadas fueron concretamente Web of Science, PsycINFO, PsycArticles, Scopus, CSIC, Psicodoc, y PubMed. Respecto a los filtros de búsqueda en las distintas bases de datos fueron aplicados los siguientes: artículos de revista, período de publicación artículos, trabajos con acceso a texto completo, idioma en inglés y español, y artículos evaluados por expertos.

Respecto a las estrategias de búsqueda, en un primer momento, se realizó una búsqueda usando las palabras clave, para ello se incluyó un asterisco (como un operador que permite buscar aquellas palabras que contengan la raíz y cualquier otro carácter), de modo que las palabras utilizadas para la búsqueda fueron: mindfulness*, ansiedad, niños*, adolescentes*, niños y adolescentes*, intervención, terapia y tratamiento*, así como, sus equivalentes en inglés (mindfulness, anxiety, child, adolescent, children and adolescent, intervention, therapy and treat). Igualmente, se realizó una nueva búsqueda, utilizando tanto el operador booleano AND, como OR, usando las mismas palabras en cada una de las bases de datos, es decir: (mindfulness*) AND (anxiety) AND (child*) OR (adolescent*) OR (children and adolescent*) AND (intervention) OR (therapy) OR (treat*). En este sentido, y debido a que en las bases de datos CSIC y Psicodoc se obtuvieron muy pocos resultados, tras la combinación de búsqueda, se amplió la búsqueda, con una nueva combinación más simple: (mindfulness) OR (conciencia plena) AND (ansiedad).

Tras dicha búsqueda, se obtuvieron un total de 2025 trabajos para revisión y lectura. El período de la búsqueda incluyó aquellos trabajos publicados desde la creación de las distintas bases de datos, hasta la fecha del mes de abril de 2019. 


\section{- CRITERIOS DE INCLUSIÓN Y EXCLUSIÓN}

Se incluyeron aquellos artículos que cumplieron con los siguientes criterios de inclusión: 1) Estudios en inglés y español; 2) que evalúen la relación entre mindfulness y ansiedad; 3) de intervención, que aporten los datos numéricos necesarios para poder realizar el metaanálisis; 4) con acceso al texto completo; 5) evaluados por expertos; 6) que utilicen el mismo diseño de estudio, en este caso, longitudinal; y, 7) que muestren un tamaño muestral de mínimo diez sujetos.

En cuanto a los criterios de exclusión establecidos fueron: 1) trabajos duplicados; 2) que sólo aporten datos cualitativos; 3) publicados en otro idioma diferente al inglés o español; 4) que guarden relación con otras cuestiones asociadas al estrés o ansiedad; 5) que no sean de intervención; 6) tesis, capítulos de libro, libros o revisiones; y, 7) que no muestren la Medias (M) y Desviaciones Típicas (DT) en el apartado resultados, en relación a pretest/postest de dicha intervención.

\section{-EXTRACCIÓN DE DATOS}

Primero, se diseñó un formulario de extracción de datos que incluía información sobre los criterios de inclusión establecidos (autores y año de publicación, diseño, muestra, variables evaluadas, país donde se realiza el estudio e instrumento utilizado). Con la ayuda de este formulario, se extrajeron datos cuantitativos en relación al tamaño de la muestra y las medidas de las variables de resultado. Este proceso fue realizado por tres revisores, asegurando la máxima fiabilidad en la recogida de información. Los casos donde hubo controversia entre los revisores, se resolvieron por consenso en reuniones, con la participación de un cuarto colaborador.

Para la selección de artículos, en primer lugar se realizó una lectura del título, así como del resumen de la población fijada, con el objetivo de aplicar el primer criterio de exclusión. Posteriormente, se realizó una lectura comprensiva del texto completo por parte de los tres miembros del equipo, para así poder aplicar el resto de criterios conceptuales y metodológicos establecidos.

\section{- RIESGO DE SESGO}

Se utilizó la herramienta de evaluación de riesgo de sesgo de Cochrane Collaborations (Higgins et al., 2011). Dos investigadores de forma independiente evaluaron el riesgo de sesgo, con el fin de resolver desacuerdos en una reunión consensuada por el investigador principal.

\section{-ANÁLISIS DE DATOS: METAANÁLISIS}

Para el metaanálisis se seleccionaron aquellos estudios que habían utilizado una intervención, es decir, un pretest-intervenciónpostest, de manera que se pudiese comprobar la eficacia de dicha intervención. En este sentido, además de lo anterior, se seleccionaron aquellos trabajos que aportaban datos numéricos, tanto en el pretest, como en el postest, referentes al tamaño de la muestra, la $M$ y la DT. Para el análisis, se seleccionó la opción Intervention Review para comprobar la efectividad de las intervenciones, utilizando un método estadístico de diferencias de medias estandarizada, puesto que los estudios seleccionados habían utilizado distintos instrumentos de medición. El sesgo de publicación asociado con el significado estadístico de la investigación no se pudo analizar gráficamente con un gráfico en embudo debido al número de estudios seleccionados (Higgins y Green 2011), ya que se necesitan un mínimo de diez trabajos (Egger, Smith, Schneider, y Minder, 1997). Se ejecutó un metaanálisis con un modelo de efectos fijos con el programa Gerente de Revisión Cochrane (Rev Man), versión 5.3., para así poder comprobar la heterogeneidad entre los diferentes estudios, el tamaño del efecto, la calidad de los datos, etc. (Sánchez-Meca y Ato, 1989).

\section{RESULTADOS}

La figura 1 muestra el proceso de selección de estudios (diagrama de flujo). Un total de 2025 trabajos fueron identificados. 357 trabajos duplicados fueron eliminados. Se revisaron un total de 1668 estudios, de los cuales, por diversos conceptos, quedaron 41 para revisión. Posteriormente, fueron eliminados 


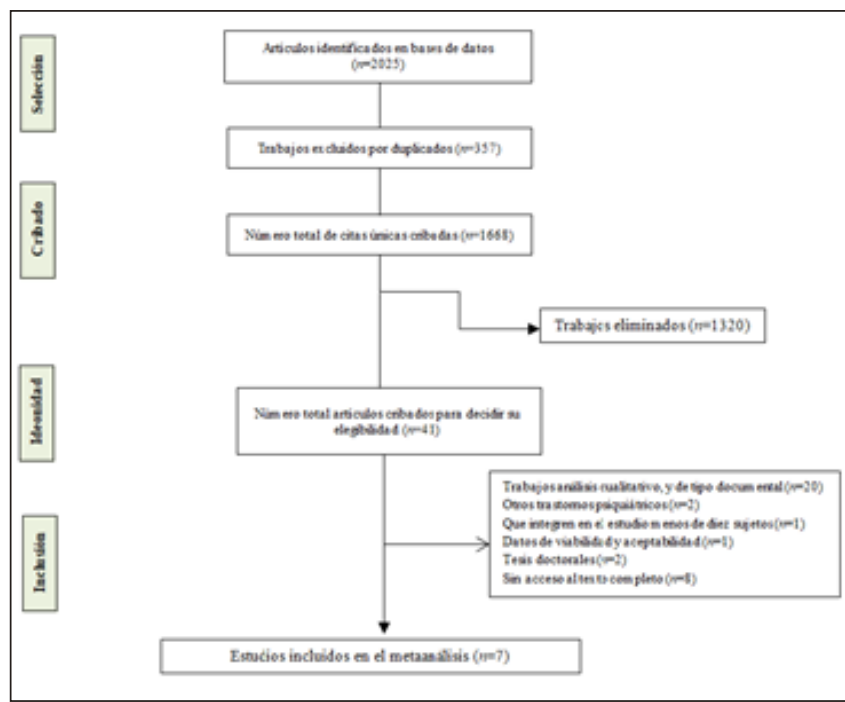

Figura 7: Diagrama de flujo con los pasos en la selección de los artículos

34 artículos por diversos motivos, tal y como se puede observar en la figura 1. Finalmente, un total de siete trabajos se incluyeron en el metaanálisis.

Los diferentes estudios llevaron a cabo una intervención hacia sus participantes, evaluando la eficacia de dicha intervención a través de las comparaciones entre los resultados del pretestpostest. Para ello, seis de los estudios utilizaron un tipo de estudio experimental, con un diseño longitudinal (Biegel et al., 2009; Cotton et al., 2016; Díaz-González et al. 2018; Liehr y Diaz, 2010; Semple et al. 2010), mientras que en uno se utilizó un tipo de estudio cuasi-experimental, con un diseño longitudinal (Cuéllar, Bazán, y Araya, 2019).

El tamaño muestral de los trabajos varió de 10 a 55 participantes. Los estudios estaban compuestos por estudiantes universitarios, en este caso, los trabajos realizados por Cuéllar, Bazán, y Araya (2019) y por Soto-Vázquez y AlvaradoGarcía (2018), por adolescentes de edades inferiores (Biegel et al., 2009), por niños (Liehr y Diaz, 2010), así como, por niños y adolescentes (Cotton et al., 2016; Díaz-González et al., 2018; Semple et al., 2010).

En relación a la composición de género de la muestra, en uno de ellos la mayoría pertenecían al género masculino, concretamente, el trabajo realizado por Cuéllar, Bazán, y Araya (2019), versus a otro estudio, en el que predominaba el género femenino (Biegel et al., 2009). Sin embargo, uno de los estudios no mostraba la composición en relación al género de los participantes (Soto-Vázquez y AlvaradoGarcía, 2018). Así pues, en cuatro trabajos sí que se presentaba una equiparación entre ambos géneros (Cotton et al., 2016; Díaz-González et al., 2018; Liehr y Diaz, 2010; Semple et al. 2010).

Respecto a las prácticas de $\mathrm{MBI}$, se muestran diferencias en cuanto al tiempo de intervención. En este sentido, en el trabajo realizado por Díaz-González et al. (2018) Ilevaron a cabo la intervención durante ocho sesiones, parecido a la intervención realizada por Soto-Vázquez y Alvarado-García (2018), que duró diez sesiones. Sin embargo, la intervención realizada por Liehr y Diaz (2010) duró dos semanas, la de Semple et al. (2010) y Biegel et al. (2009) ocho semanas, la de Cuéllar, Bazán, y Araya (2019) once semanas, mientras que la de Cotton et al. (2016) duró doce semanas.

En cuanto a los resultados de los estudios seleccionados, tal y como se puede observar en la tabla 1, se exponen los resultados obtenidos para el análisis de la relación entre mindfulness y ansiedad, en los que han participado un total de 189 sujetos. En consecuencia, para extraer la información de este trabajo los estudios fueron codificados siguiendo el siguiente proceso: (1) Autores y año de la publicación; (2) Tipo de estudio/ procedimiento empleado en la metodología; (3) País; (4) Variable evaluada; (5) Muestra; e, (6) Instrumento utilizado en la intervención.

\section{- RESUMEN DE RESULTADOS: METAANÁLISIS}

Se excluyeron un total de dos artículos, concretamente, uno de ellos, por corresponderse con una tesis doctoral, quedando eliminado tras aplicar uno de los criterios de exclusión establecidos. El segundo trabajo excluido por no mostrar en el apartado resultados las M, así como las DT, concretamente, fue el estudio realizado por Strawn et al. (2016). En este sentido, el metaanálisis se realizó para la variable estrés, quedando incluidos en el mismo un total de siete trabajos.

\section{-VARIABLE DE RESULTADO ANSIEDAD}

Siete fueron los estudios seleccionados para la variable ansiedad, con un total de 189 
Tabla 1

Conjunto de estudios que tratan la relación entre estrés y ansiedad

\begin{tabular}{|c|c|c|c|c|c|}
\hline $\begin{array}{l}\text { Autores y Año } \\
\text { publicación }\end{array}$ & $\begin{array}{l}\text { Tipo de estudio/ } \\
\text { procedimiento }\end{array}$ & Lugar & $\begin{array}{l}\text { Variables } \\
\text { evaluadas }\end{array}$ & Muestra & $\begin{array}{l}\text { Instrumento evaluación } \\
\text { ansiedad }\end{array}$ \\
\hline $\begin{array}{l}\text { Cuéllar, Bazán, y Araya } \\
\text { (2019) }\end{array}$ & $\begin{array}{l}\text { Experimental, con } \\
\text { mediciones pre y } \\
\text { post tratamiento }\end{array}$ & $\begin{array}{l}\text { Costa } \\
\text { Rica }\end{array}$ & $\begin{array}{l}\text { Conciencia de } \\
\text { atención plena y } \\
\text { ansiedad }\end{array}$ & 12 & $\begin{array}{c}\text { Hamilton Anxiety Scale [HAS] (Hamilton, } \\
\text { 1959), en su versión en español } \\
\text { elaborada por Carrobles, Costa, Del } \\
\text { Ser, y Bartolomé (1987) }\end{array}$ \\
\hline $\begin{array}{l}\text { Biegel, Shapiro, Brown, } \\
\text { y Schubert (2009) }\end{array}$ & $\begin{array}{l}\text { Diseño factorial } \\
\text { mixto, con } \\
\text { mediciones pre y } \\
\text { post tratamiento }\end{array}$ & México & $\begin{array}{l}\text { Conciencia plena, } \\
\text { salud mental, } \\
\text { síntomas de } \\
\text { angustia, ansiedad, } \\
\text { estrés y autoestima }\end{array}$ & 33 & $\begin{array}{l}\text { The State-Trait Anxiety Inventory for } \\
\text { Children [STAl-C] (Spielberger, 1973) }\end{array}$ \\
\hline $\begin{array}{l}\text { Díaz-González, Pérez, } \\
\text { Sánchez-Raya, } \\
\text { Moriana, y Sánchez } \\
\text { (2018) }\end{array}$ & $\begin{array}{l}\text { Controlado } \\
\text { aleatorio, con } \\
\text { mediciones pre y } \\
\text { post tratamiento }\end{array}$ & España & $\begin{array}{c}\text { Conciencia plena, } \\
\text { estrés, ansiedad, } \\
\text { autoestima y salud } \\
\text { mental }\end{array}$ & 41 & $\begin{array}{l}\text { The State-Trait Anxiety Inventory for } \\
\text { Children [STAl-C] (Spielberger, 1973) }\end{array}$ \\
\hline $\begin{array}{l}\text { Soto-Vázquez y } \\
\text { Alvarado-García (2018) }\end{array}$ & $\begin{array}{l}\text { Experimental, con } \\
\text { mediciones pre y } \\
\text { post tratamiento }\end{array}$ & Perú & $\begin{array}{l}\text { Ansiedad y estrés } \\
\text { académico }\end{array}$ & 55 & $\begin{array}{l}\text { Anxiety Inventory for Children } \\
\text { (Speillberger, Gorsuch, y Lushene, 1970) }\end{array}$ \\
\hline Cotton et al. (2016) & $\begin{array}{l}\text { Seguimiento } \\
\text { controlado, con } \\
\text { mediciones pre y } \\
\text { post tratamiento }\end{array}$ & Cincinnati & $\begin{array}{l}\text { Ansiedad, } \\
\text { emociones, } \\
\text { regulación } \\
\text { emocional, } \\
\text { conciencia plena e } \\
\text { ideación suicida }\end{array}$ & 10 & $\begin{array}{l}\text { The State-Trait Anxiety Inventory for } \\
\text { Children [STAI-C] (Spielberger, 1973) }\end{array}$ \\
\hline Liehr y Diaz (2010) & $\begin{array}{l}\text { Experimental, con } \\
\text { mediciones pre y } \\
\text { post tratamiento }\end{array}$ & Florida & $\begin{array}{c}\text { Humor y } \\
\text { sentimientos cortos, } \\
\text { y ansiedad }\end{array}$ & 17 & $\begin{array}{c}\text { Anxiety Inventory for Children } \\
\text { (Speillberger, Gorsuch, y Lushene, 1970) }\end{array}$ \\
\hline $\begin{array}{l}\text { Semple, Lee, Rosa, y } \\
\text { Miller (2010) }\end{array}$ & $\begin{array}{l}\text { Seguimiento } \\
\text { controlado, con } \\
\text { mediciones pre y } \\
\text { post tratamiento }\end{array}$ & California & $\begin{array}{l}\text { Comportamiento y } \\
\text { ansiedad }\end{array}$ & 21 & $\begin{array}{l}\text { The State-Trait Anxiety Inventory for } \\
\text { Children [STAI-C] (Spielberger, 1973) }\end{array}$ \\
\hline
\end{tabular}

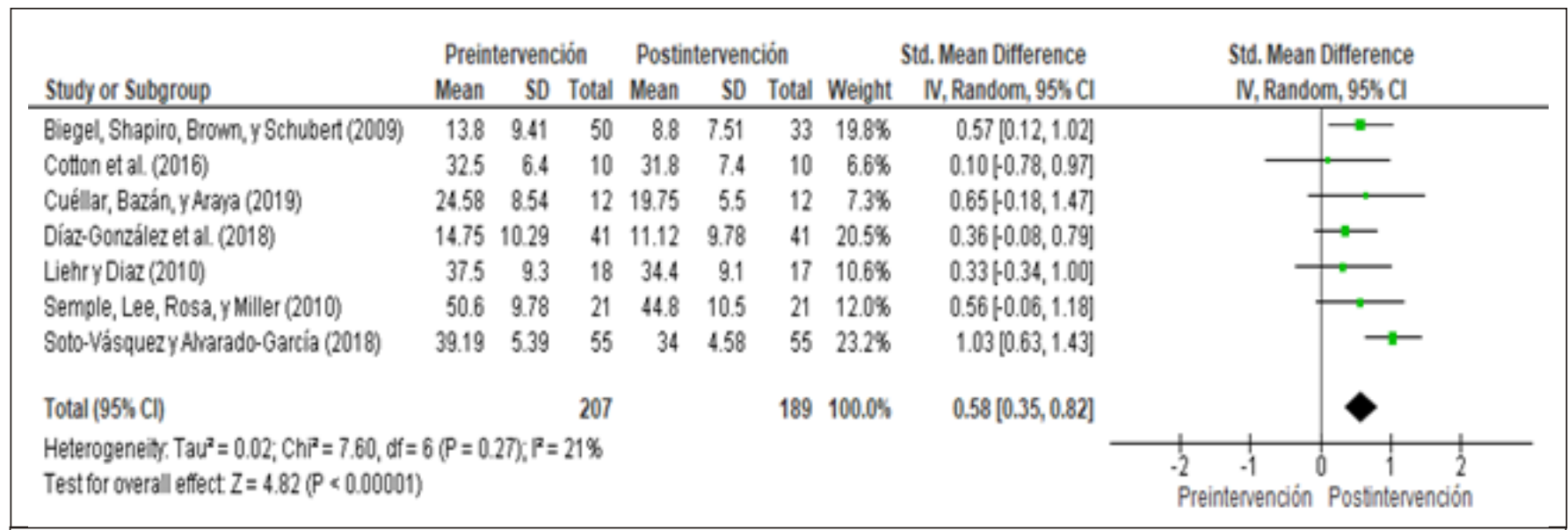

Figura 2: Diferencia de medias para variable ansiedad

participantes, donde utilizaron la ansiedad como una variable de intervención (ver Figura 2). Así pues, en seis estudios se observó un efecto positivo tras la intervención, lo que indica una clara mejoría de las intervenciones basadas en conciencia plena en la reducción de la ansiedad, mientras que, sólo en uno de ellos, no se hallaron cambios.
El promedio de la diferencia estandarizada de medias fue de $0.58(0.35,0.82)$, significativa $(Z=4.82 ; p<.00001)$. En concreto, este tamaño del efecto es mayor que el \% (PSte = .3120 ) de todos los posibles con efectos positivos (Vilariño, Amado, Vázquez, y Arce, 2018). Respecto a la heterogeneidad entre los diferentes trabajos, el $I^{2}$ mostró una heterogeneidad alta (I $=21 \%$ ), en este sentido debemos ser cautelosos 
al interpretar este resultado, ya que indica que hay moderadores de los resultados, esto puede deberse a la intervención utilizada.

\section{-RIESGO DE SESGO VARIABLE ANSIEDAD}

El riesgo de sesgo según la herramienta Cochrane de evaluación del riesgo de sesgo no fue alto, tal y como se puede visualizar en la figura 3, pudiéndose distinguir los efectos producidos entre los diferentes aspectos de cegamiento, como sería el caso de los participantes.

\section{-MODELO DE REGRESIÓN LINEAL}

El modelo de regresión lineal ha permitido visualizar la relación lineal entre el efecto de la intervención y la variable explicativa (ansiedad), tal y como se muestra en la figura 4 .

\section{DISCUSIÓN}

\section{-RESUMEN DE EVIDENCIA}

El objetivo de este trabajo consiste en identificar la relación existente entre los beneficios de la atención plena (mindfulness) y su influencia en los niveles de ansiedad en niños y adolescentes. En este sentido, Cuéllar, Bazán, y Araya (2019), examinaron el efecto de la práctica del aikido y el $\mathrm{MBI}$ sobre el estado de ansiedad, encontrando una relación positiva entre ambas intervenciones, y además de verse reducidos los niveles de ansiedad de sus participantes (Semple et al., 2010), también se hallaron variaciones positivas

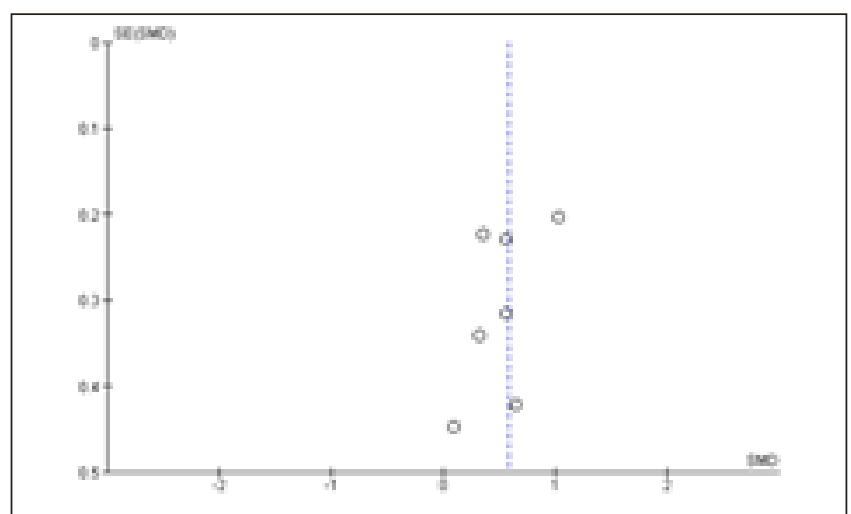

Figura 3: Riesgo de sesgo respecto al estado anímico (Lothes, Hakan, y Kassab, 2013). Pues, la práctica de conciencia plena conlleva a una reducción de emociones negativas (Tapper, 2017), de la mejora de la atención (Larrubia, 2019), así como, de hábitos de alimentación saludables en niños y adolescentes (Warren, Smith, y Ashwell, 2017), ya que una alimentación sana, así como la calidad del sueño, se ha visto relacionado con diversos factores que actúan como mediadores para hacer frente al estrés y para combatir la ansiedad (Gázquez et al., 2019).

Por su parte, Biegel et al. (2009), indican la efectividad del $\mathrm{MBl}$, puesto que vieron reducidos los síntomas de somatización, depresión y de ansiedad en sus participantes, al igual que los resultados hallados en otras líneas de investigación (Foster et al., 2018). No obstante, cuando las personas comienzan a experimentar prácticas de $\mathrm{MBI}$ se ven reflejadas en sus actividades diarias, viéndose disminuido su nivel de atención plena hasta que los efectos de la intervención se generalicen a la vida diaria (Díaz-González et al., 2018). Otros estudios realizados, en este caso con adultos, también han informado que los beneficios de la presencia plena se asocian positivamente con la cantidad de práctica, puesto que es esencial que se realicen ejercicios en casa para este tipo de intervenciones (Roselló, Zayas, y Lora, 2016).

El ambiente académico constituye una fuente de estresores que pueden dar origen a estados de ansiedad y estrés, derivando en un perjuicio para la salud, pues el discente presenta dificultades hacia el estudio, pudiendo finalizar en abandono académico. Es por ello

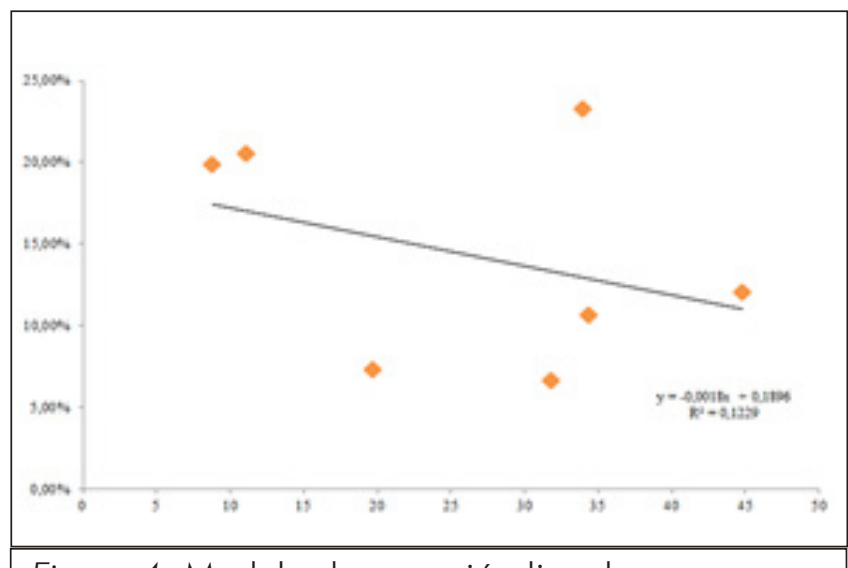

Figura 4: Modelo de regresión lineal 
que, existen intervenciones en $\mathrm{MBI}$ donde se ven reducidos la ansiedad y el estrés (Soto-Vázquez y Alvarado-García, 2018), así como, los niveles de depresión (Redstone, 2015). No podemos olvidar que, reducir la ansiedad a través del mindfulness puede disminuir la necesidad de tratamientos farmacológicos, siendo dichos fármacos mal tolerados por parte de la población joven, ya que causan síntomas peores (Cotton et al., 2016), mientras que la práctica de atención plena no perjudica la salud (Strawn et al., 2014), sino que favorece la calidad de vida del sujeto, independientemente de su edad, eliminando por ejemplo, los sentimientos de tristeza (Liehr y Diaz, 2010). En este sentido, el entrenamiento de atención plena conlleva a un incremento de la conciencia respecto a estímulos emocionales, socialmente relevantes, pudiendo disminuir la vulnerabilidad hacia la depresión (Sanger, Thierry, y Dorjee, 2018).

Tras el metaanálisis se ha podido comprobar la eficacia que ejercen las diferentes intervenciones en sus participantes, ya que la mayoría de los trabajos obtuvieron resultados favorables tras sus intervenciones. En relación a la selección aleatoria de los tamaños del efecto, indicaron un resultado positivo. Respecto al sesgo, en nuestro trabajo ha sido bajo, y en cuanto al modelo de regresión lineal, se ha podido comprobar una trayectoria ascendiente en relación a las distintas intervenciones.

Por tanto, es relevante el papel que ejercen los programas de intervención $\mathrm{MBI}$ para reducir los niveles de ansiedad, tal y como indican Gallego et al. (2016). Pues, se ha observado que los programas de atención plena ayudan a responder más adecuadamente a aquellos estresores producidos en el contexto laboral (Kemper, Mo, y Khayat, 2015), además de reducir los niveles de ansiedad (Strawn et al., 2016) y prevenir el síndrome de burnout (Virgili, 2015). En definitiva, los programas de intervención reducen de manera notable los síntomas depresivos, favorecen el uso de estrategias de afrontamiento encaminadas a la resolución eficaz de problemas, permiten la mejora del estado de salud psicofísica, además de favorecer la respuesta de conductas asertivas ante las diversas situaciones vivenciales (Roldán et al., 2015). En este sentido, aquellas personas que practican mindfulness aprenden a ser más conscientes de sus pensamientos (LópezGonzález et al., 2018; Rawlett, Friedmann, y Thomas, 2019), así como de sus sentimientos y sensaciones corporales a medida que avanza la intervención (Duarte y Pingo-Gouveia, 2016), mostrando consigo un mayor bienestar (Barrera-Herrera et al., 2019) y una mejora en lo que respecta a su calidad de vida (Giménez y Tortosa, 2018).

\section{CONCLUSIONES}

El presente trabajo aporta tras la revisión de los distintos estudios que la ansiedad guarda relación con la salud psicofísica del sujeto. En este sentido, cuando se presentan niveles bajos en ansiedad se muestra un incremento en relación al bienestar personal.

Los programas de intervención que implican una práctica prolongada en el tiempo permiten reducir los trastornos de ansiedad. Es por ello que deben tenerse en cuenta el diseño de técnicas y programas de intervención duradera, ya que favorecen hacia una mayor eficacia tras sus intervenciones.

Entre las limitaciones de este trabajo se encuentran, por un lado, la estrategia de búsqueda empleada, puesto que la estrategia de búsqueda del presente trabajo se ha centrado en las bases de datos Web of Science, PsycINFO, PsycArticles, Scopus, CSIC, Psicodoc, y PubMed, por lo que es posible que se haya podido omitir de manera involuntaria trabajos publicados en otros recursos. Por otro lado, se encuentra la falta de datos cuantitativos en el apartado resultados de distintos estudios, lo que no ha permitido incluirlos en el presente trabajo por su relación con el metaanálisis.

Como futura línea de investigación es aconsejable para ampliar el conocimiento acerca de este tema ofrecer el diseño de nuevos estudios longitudinales, de manera que a partir de los mismos se puedan realizar nuevas comparaciones y poder extraer nuevas conclusiones más sólidas. En este sentido, se sugiere que nuevas líneas de investigación incluyan sus resultados cuantitativos más relevantes en sus trabajos, de manera que permitan que se lleven a cabo nuevas 
investigaciones futuras centradas en esta misma línea.

Las implicaciones de este trabajo son destacadas, ya que la intervención a través de este tipo de programas ejerce un papel relevante en lo que respecta a la calidad de vida de la persona. Por tanto, dada la cercanía, sencillez y disponibilidad de programas para dominar técnicas de mindfulness se sugiere ponerlas en práctica, puesto que permitirían potenciar la salud psicofísica de niños y adolescentes, independientemente del contexto del que formen parte.

\section{- Agradecimientos.}

El presente estudio cuenta con la colaboración de la Excma. Diputación Provincial de Almería. Parte de este trabajo se ha desarrollado gracias a la Ayuda para Contratos Predoctorales para la formación de Doctores (PRE2018-085434) concedida a José Gabriel Soriano Sánchez dentro del proyecto con Referencia: EDU2017-88139-R financiado por el Programa Estatal de Investigación, Desarrollo e Innovación Orientada a los Retos de la Sociedad, en el marco del Plan Estatal de Investigación Científica y Técnica y de Innovación, y la cofinanciación con Fondos Estructurales de la Unión Europea.

- Conflicto de intereses.

Los autores declaran no tener ningún conflicto de intereses.

\section{REFERENCIAS}

Alarcón, P.A., Pérez-Luco, R.X., Wenger, L.S., Salvo, S.I., y Chesta, S.A. (2018). Personalidad y gravedad delictiva en adolescentes con conducta antisocial persistente. Revista Iberoamericana de Psicología y Salud, 9(1), 58-74. doi: 10.23923/i.rips.2018.01.015

Álvarez-García, D., Núñez, J.C., García, T., y Barreiro-Collazo, A. (2018). Individual, Family, and Community Predictors of Cyber-aggression among Adolescents. The European Journal of Psychology Applied to Legal Context, 10(2) 7988. doi: doi.org/10.5093/ejpalc2018a8

Amutio, A., Franco, C., Sánchez, L.C., PérezFuentes, M.C., Gázquez, J.J., Van Gordon, W., y Molero, M.M. (2018). Effects of Mindfulness Training on Sleep Problems in Patients With Fibromyalgia. Frontiers in Psychology, 9, 1-8. doi: 10.3389/fpsyg.2018.01365

Barrera-Herrera, A., Neira-Cofré, M.J., RaipánGómez, P., Riquelme-Lobos, P., y Escobar,
B. (2019). Apoyo social percibido y factores sociodemográficos en relación con los síntomas de ansiedad, depresión y estrés en universitarios chilenos. Revista de Psicopatología y Psicología Clínica, 24, 105-115. doi: 10.5944/ rppc. 23676

Biegel, G.M., Brown, K.W., Shapiro, S.L., y Schubert, C.M. (2009). Mindfulness-based stress reduction for the treatment of adolescent psychiatric outpatients: a randomised clinical trial. Journal of Consulting and Clinical Psychology, 77, 855-866. doi: 10.1037/ a0016241

Bishop, S.R., Lau, M., Shapiro, S., Carlson, L., Anderson, N.D., y Carmody, J. (2004). Mindfulness: A proposed operational definition. Clinical Psychology: Science \& Practice, 11, 230-24 1. doi: 10.1093/clipsy.bph077

Carrobles, I., Costa, C.M., Del Ser, T., y Bartolomé, P. (1987). La práctica de la terapia de conducta: teoría y método de aplicación para la práctica clínica. Valencia: Promolibro.

Cotton, S., Luberto, C.M., Sears, R.W., Strawn, J.R., Stah, L., Wasson, R.S. Blom, T.J., y Delbello, M.P. (2016). Mindfulness-based cognitive therapy for youth with anxiety disorders at risk for bipolar disorder: a pilot trial. Early Intervention in Psychiatry, 10, 426-434. doi: 10.1111 /eip. 12216

Cuéllar, R., Bazán, A., Araya, G.A. (2019). Efectos de la práctica de aikido sobre mindfulness y la ansiedad en estudiantes universitarios de Costa Rica. Retos, 35, 13-19. doi: 10.14589/ ido.14.2.6.

Díaz-González, M.C., Pérez, C., Sánchez-Raya, A., Moriana, J.A., y Sánchez, V. (2018). Mindfulnessbased stress reduction in adolescents with mental disorders: A randomised clinical trial. Psicothema, 30(2), 165-170. doi: 10.7334/ psicothema2017.259

Duarte, J., y Pinto-Gouveia, J. (2016). Effectiveness of a mindfulness-based intervention on oncology nurses' burnout and compassion fatigue symptoms: A nonrandomized study. International Journal of Nursing Studies, 64, 98-107. doi: 10.1016/i.ijnurstu.2016.10.002 Duncan, G.J., Dowsett, C.J., Claessens, A., Magnuson, K., Huston, A.C., y Klebanov, P. 
(2007). School readiness and later achievement. Developmental Psychology, 43, 1428-1446. doi: 10.1037/0012-1649.43.6.1428

Egger, M., Smith, G.D., Schneider, M., y Minder, C. (1997). Bias in meta-analysis detected by a simple graphical test. British Medical Journal, 315, 629-634. doi: 10.1136/ bmi.315.7109.629

Ehrenreich, J.T., y Gross, A.M. (2002). Biased attentional Behavior in childhood anxiety a review of theory and current empirical investigation. Clinical Psychology Review, 22, 991-1008. doi: 10.1016/S0272-7358(01)00123-4

Foster, B., Lomas, J., Downey, L., y Stougch, C. (2018). ¿̇a inteligencia emocional media la relación entre la atención plena y la ansiedad y la depresión en los adolescentes? Psicología Conductual, 9, 2483.

Gallego, J., Aguilar-Parra, J.M., Cangas, A.J., Rosado, A., y Langer, A.I. (2016). Efecto de intervenciones mente/cuerpo sobre los niveles de ansiedad, estrés y depresión en futuros docentes de Educación Primaria: un estudio controlado. Revista de Psicodidáctica, 21 (1), 87-101. doi: 10.1387/RevPsicodidact.13256

Gázquez, J.J., Pérez-Fuentes, M.C., Carrión, J.J., Luque, A., y Molero, M.M. (2015). Interpersonal Value profiles and analysis to adolescent behavior and social attitudes. Revista de Psicodidáctica, 20(2), 321 -337. doi: 10.1387/RevPsicodidact. 12978

Gázquez, J.J., Pérez-Fuentes, M.C., Molero, M.M., Barragán, A.B., Martos, A. , y Sánchez-Marchán, C. (2016). Drug use in adolescent in relation to social support and reactive and protective aggresive behaviour. Psicothema, 28(3), 318 322. doi: 10.7334/psicothema2015.327

Gázquez, J.J., Pérez-Fuentes, M.M., Molero, M.M., Oropesa, N.F., Simón, M.M., y Saracostti, M. (2019). Sleep Quality and the Mediating Role of Stress Management on Eating by Nursing Personnel. Nutrients, 17 (8), 1-10. doi: 10.3390/nu 11081731

Giménez, J., y Tortosa, J. (2018). Beneficios del ejercicio físico para la salud mental en pacientes drogodependientes. Revista Española de Drogodependencia, 43(2), 62-74.

Guerra, C., y Barrera, P. (2017). Psicoterapia con víctimas de abuso sexual inspirada en la terapia cognitivo-conductual centra en el trauma. Revista de Psicología, 26(2), 1 - 13, doi: $\underline{10.5354 / 0719-0581.2017 .47952}$

Hamilton, M. (1959). The assessment of anxiety states by rating. British Journal of Medicine and Psychology, 32(1), 50-55. doi: 10.1111/ j.2044-8341.1959.tb00467.x

Higgins, J.P., y Green, S. (2011). Cochrane Handbook for Systematic Reviews of Interventions Version 5.1.0. [Cited 15 September 2018]. Recuperado de: http:// handbook.cochrane.org

Higgins, J.P.T., Altman, D.G., Gøtzsche, P.C., Juni, P., Moher, D., Oxman, A.D.,...Cochrane Statistical Methods Group (2011). The Cochrane Collaboration's tool for assessing risk of bias in randomised trials. British Medical Journal, 343, d5928. doi: 10.1136/bmi. d5928

Ho, S.M.Y., Tong, D.W., Mak, C., y Kei, K.W. (2018). Cognitive factors associated witg depressión and anxiety in adolescents: A twoyear longitudinal study. International Journal of Clinical and Health Psychology, 18, 227-234. doi: 10.1016/i.iichp.2018.04.001

Joiner, T.E., Steer, R.A., Beck, A.T., Schmidt, N.B., Rudd, M.D., y Catanzaro, S.J. (1999). Physiological hyperarousal: Construct validity of a central aspect of the tripartite model of depression and anxiety. Journal of Abnormal Psychology, 108, 290-298. doi: 10.1037/0021-843X.108.2.290

Kemper, K.J., Mo, X., y Khayat, R. (2015). Are mindfulness and self-compassion associated with sleep and resilience in health professionals? The Journal of Alternative and Complementary Medicine, 21, 496-503. doi: 10.1089/ acm.2014.0281

Larrubia, A. (2019). MBI (Mindfulness Based Intervention). Desarrollo profesional y salud laboral. Revista de Investigación y Educación en Ciencias de la Salud, 4, 4-27.

Liehr, P., y Diaz, N. (2010). A pilot study examining the effect of mindfulness on depression and anxiety for minority children. Archives of Psychiatric Nursing, 24, 69-71. doi: 10.1016/i. apnu.2009.10.001 
López-González, L., Amutio, A., Oriol, X., Gázquez, J.J., y Pérez-Fuentes, M.C. (2018). Development and validation of the Relaxation-Mindfulness Scale for Adolescents (EREMIND-A). Psicothema, 30(2), 224-231. doi: 10.7334/psicothema2017.225

Lothes II, J., Hakan, R., y Kassab, K. (2013). Aikido experience and its relation to mindfulness: a twopart study. Perceptual and Motor Skills, 1 16(1), 30-39. doi:10.2466/22.23.PMS.1 16.1.30-39

Lutz, J., Brühl, A.B., Scheerer, H., Jäncke, L., y Herwig, U. (2016). Neural correlates of mindful self-awareness in mindfulness meditators and meditation-naïve subjects revisited. Biological Psychology, 119 (2016), 21 -30. doi: 10.1016/i.biopsycho.2016.06.01

Macías, J.J., Valero-Aguayo, L., Bond, F.W., y Blanca, M.J. (2019). The efficacy of functionalanalytic psychotherapy and Acceptance and commitment therapy (FACT) for public employees. Psicothema, 31(1), 24-29. doi: 10.7334/psicothema2018.202

Moher, D., Liberati, A., Tetzlaff, J., y Altman, D.G. (2009). Preferred reporting items for systematic reviews and meta-analyses: the PRISMA Statement. BMJ, 339.

Peña, E., Bernal, L., Pérez, R., Reyna, L., y García, K.G. (2017). Stress and coping strategies in Higher Level Students of the Autonomous University of Guerrero. NURE Investigación, 15(92), 1-8.

Pérez-Fuentes, M.C., Gázquez, J.J., Molero, M.M., Cardila, F., Martos, A., Barragán, A.B.,... Mercader, I. (2015). Impulsividad y consumo de alcohol y tabaco en adolescentes. European Journal of Investigation in Health, 5(3), 371382. doi: 10.1989/ejihpe.v5i3.139

Pérez-Fuentes, M.C., Molero, M.M., Carrión, J.J., Mercader, I., y Gázquez, J.J. (2016). Sensationseeking and impulsivity as predictors of reactive and proactive aggression in adolescents. Frontiers in Psychology, 7, 1-8. doi: 10.3389/ fpsyg.2016.01447

Rawlett, K.E., Friedmann, E., y Thomas, S.A. (2019). Intervención basada en la atención plena con un grupo de comparación atencional en adolescentes en riesgo: un ensayo piloto aleatorizado y controlado. Integrative Medicine Research, 8(2), 101-106. doi: 10.1016/i.

\section{imr.2019.04.002}

Redstone, L. (2015). Mindfulness Meditation and Aromatherapy to Reduce Stress and Anxiety. Archives of Psychiatric Nursing, 29(3), 192203. doi: 10.1016/i.apnu.2015.03.001

Roldán, G.M., Salazar, I.C., Garrido, L., y Cuevas-Toro, A.M. (2015). Intervención psicológica grupal, basada en la aceptación y el entrenamiento en comunicación, con familiares de personas con trastorno mental grave. Anales de Psicología, 31 (3), 759-772. doi: 10.6018/analesps.31.3.154071

Roselló, J., Zayas, G., y Lora, V. (2016). Impacto de un adiestramiento en meditación en conciencia plena (mindfulness) en medidas de ansiedad, depresión, ira y estrés y conciencia plena: un estudio piloto. Revista Puertorriqueña de Psicología, 27(1), 62-78.

Sánchez-Meca, J., y Ato, M. (1989). Metaanálisis: Una alternativa metodológica a las revisiones tradicionales de la investigación. En J. Arnau y H. Carpintero (Eds.), Tratado de psicología general I: Historia, teoría y método (pp. 617 669). Madrid: Alhambra.

Sanger, K.L., Thierry, G., y Dorjee, D. (2018). Efectos del entrenamiento del mindfulness en la escuela sobre el procesamiento de las emociones y el bienestar en los adolescentes: evidencia de potenciales relacionados con eventos. Developmental Science, 21 (5), e12646. doi: $10.1111 /$ desc. 12646

Semple, R.J., Lee, J., Rosa, D., y Miller, L.F. (2010). $A$ randomized trial of mindfulness-based cognitive therapy for children: promoting mindful attention to enhance socialemotional resiliency in children. Journal of Child and Family Studies, 19, 218-229. doi: 10.1007/ s10826-009-9301-y

Soto-Vázquez, M.L., y Alvarado-García, P.A. (2018). Efecto del aceite esencial de Peperomia dolabriformis y meditación mindfulness en niveles de ansiedad y estrés académico de estudiantes universitarios. Medicina Naturista, 12(1), 9-14.

Spielberger, C.D. (1973). State-Trait Anxiety Inventory for Children: Preliminary manual. Palo Alto, CA: Consulting Psychologists Press.

Spielberger, C.D., Gorsuch, R.L., y Lushene, R.E. (1970). Manual for the State-Trait 
Anxiety Inventory. Palo Alto, CA: Consulting Psychologists Press.

Strawn, J.R., Adler, C.M., McNamara, R.K., Welge, J.A., Bitter, S.M., Mills, N.P.,...Del Bello, M.P. (2014). Antidepressant tolerability in anxious and depressed youth at high risk for bipolar disorder: a prospective naturalistic treatment study. Bipolar Disord, 16(5), 523-530. doi: $10.1111 /$ bdi. 12113

Strawn, J.R., Cotton, S., Luberto, C.M., Patino, L.R., Stahl, L.A., Weber, W.A.,...Del Bello, M.P. (2016). Neural function before and after mindfulness based cognitive therapy in anxious adolescents at risk for developing bipolar disorder. Journal of Child and Adolescent Psychopharmacology, 26, 1-8. doi: 10.1089/ cap.2015.0054

Tapper, K. (2017). Can mindfulness influence weight management related eating behaviors? If so, how? Clinical Psychology Review, 53(Suppl. C), 122-134. doi: 10.1016/i. cpr.2017.03.003

Tylka, T.L. (2006). Development and psychometric evaluation of a measure of intuitive eating. Journal of Counseling Psychology, 53, 226240. doi: 10.1037/0022-0167.53.2.226

Vallejo, P.M.A. (2006). Mindfulness. Papeles del Psicólogo, 27(2), 92-99.

Vilariño, M., Amado, B. G., Vázquez, M. J., y Arce, R. (2018). Psychological harm in women victims of intimate partner violence: Epidemiology and quantification of injury in mental health markers. Psychosocial Intervention, 27(3), 145152. doi: $10.5093 /$ pi2018a23
Virgili, M. (2015). Mindfulness-based interventions reduce psychological distress in working adults: A meta-analysis of intervention studies. Mindfulness, 6, 326-337. doi: 10.1007/ s12671-013-0264-0

Warren, J.M., Smith, N., y Ashwell, M. (2017). A structured literature review on the role of mindfulness, mindful eating and intuitive eating in changing eating behaviours: Effectiveness and associated potential mechanisms. Nutrition Research Reviews, 30, 272-283. doi: 10.1017/ S0954422417000154

Zeidan, F., y Vago, D.R. (2016). Mindfulness meditation-based pain relief: a mechanistic account. Annals of the New York Academy of Sciences, 1373(1), 114-127. doi: 10.1111/ nyas. 13153

Zuñeda, A., Yamazares, A., Marañón, D., y Vázquez, G. (2016). Características individuales y familiares de los adolescentes inmersos en violencia filio-parental: La agresividad física, la cohesión familiar y el conflicto interparental como variables explicativas. Revista de Psicopatología y Psicología Clínica, 21, 21-33. doi: 10.5944/rppc.vol.21.num.1.2016.15021 